\title{
Comparative Study of Tumor Detection Techniques with their Suitability for Brain MRI Images
}

\author{
Deepak Baghel \\ Department of Electronics and Communication \\ Technology, \\ Samrat Ashok Technological Institute, \\ Vidisha, 464001, India
}

\author{
K.G. Kirar \\ Assistant Professor, Department of Electronics and \\ Instrumentation, \\ Samrat Ashok Technological Institute, \\ Vidisha, 464001, India
}

\begin{abstract}
This paper is a study of detection of Brain tumor in MRI images by using simple Canny Edge Detection Technique , canny technique and Fuzzy c-means method by using Morphological Operations. The canny edge detection technique defines edges of the MRI image by using many parameter like thresholding, thinning etc. canny with morphological operation like dilation, erosion etc., where simply applied on it for getting better results, and fuzzy c-means method gives best results for segmentation of Brain tumor in MRI images. Segmentation is very important task for detection of area of intrest; after the segmentation morphological operation are applied to detect tumor in MRI brain images, these methods are tested over multiple MRI tumorous and nontumorous images.
\end{abstract}

\section{General terms}

Edge based Segmentation, clustering

\section{Keywords}

MRI Brain Images, Segmentation, Canny Edge Detection Technique, Morphological Operations, Fuzzy c-means method.

\section{INTRODUCTION}

Image processing has broad area in terms of applications and image processing is most frequently used in the medical science, agriculture field, remote sensing and various other fields. The medical science technology most commonly uses image processing in diseases like cancer tumor detection. This paper perpose algorithm to process MRI or CT scanning system images to detect Brain tumor. MRI (magnetic resonance imaging) is the most widely used technology because of its high quality performance, it gives better quality of result compared to any other method, especially in brain imaging. Segmenting MRI brain image for extracting tumor or any other region of an organ requires knowledge of brain anatomy. Developing brain tumor extraction software relieves radiologists from the task of identifying a region of interest but for reliable segmentation results it should incorporate some improvements by an expert.

The canny edge detection method is best known for its edge detection because this method gives us better result compared to any other edge detection technique, So canny method uses for the edge detection technique in MRI image for the detection of tumor. In canny edge detection technique edge detection is done in both the direction ( $\mathrm{X}$ and $\mathrm{Y}$ ), after that norm of them are taken and then thresholding of that norm is done, at last thinned of the image is done and result came in terms of edges. Enhance the canny result by applying a morphological operation.

This paper concentrate on the best method of detection of Brain tumor which is Fuzzy c-means clustering algorithm[2,3,4], In the fuzzy c-means algorithm clusters can formally be seen as subset of data set, one possible classification of clustering methods can be according to whether the subsets are fuzzy or crisp, fuzzy segmentation of brain MRI images plays an important role. Segmentation of image is done by using a fuzzy clustering algorithm which is well known as a soft segmentation algorithm which keeps more information from input image than hard segmentation method. K-means clustering, an unsupervised method produces hard segmentation by restricting a pixel's membership exclusive to one class. K-means is suitably known for MIS as the number of clusters is usually known for images of a particular region of human anatomy. K-means has been used extensively for segmentation of MRI images. Morphology is used to transform an image into another image by eliminating undesirable features[8-11]. This is done by probing the input image with another image of certain shape and size known as structuring element.

\section{METHODOLOGY}

Already there is a lot of work which has been done in the area of brain tumor detection. For the detection of tumor CT (computed tomography) or MRI (magnetic resonance imaging) images are uses.In preposed methodology MRI images are used. Segmentation plays most important role in detecting the brain tumor. There are three best methods which are used to detected tumor,

- Canny edge detection technique.

- Canny edge detection method with using morphological operations.

- Fuzzy c-means method by using morphological operations.

Edge detection also a segmentation technique. Basically Edge defines as; where the vertical and the horizontal surfaces of an object meet. The edge may be not look like an edge any more in an image taken on the ground. If there were sensor with extensively small footprints and zero-width point spread functions, an edge would be recorded between pixels within an image. Edges are scale-dependent and an edge may contain other edges, but at a certain scale, an edge still has no width Edges characterize boundaries and a problem of fundamental importance in image processing. Edges in images are areas with strong intensity contrasts a jump in intensity from one pixel to the next. Edge detecting an image significantly reduces the amount of data and filters out excessive information, while keeping the important structural properties in an image.

\subsection{Canny edge detection technique}

Marr and Hildreth previously worked on the canny edge detector. The perpouse of edge detection in general is to significantly reduce the amount of data in an image. Several egde detection technique already exist but the canny edge detection technique is best suited for our perception it is also an old technique but it is 
an standard edge detection technique. It is widely used in current image processing techniques with further improvements.

The Canny edge detection algorithm is known as the optimal edge detector. Cranny's intentions had been to enhance the many edge detectors already out at the time. There are some basic criterion on which Canny edge detection is done; The first is low error rate. It is important that edges occurring in images should not be missed and NO responses to non-edges. The second criterion is that the edge points are well localized that is the distance between the edge pixels as found by the detector and the actual edge is to be at a minimum. The third criterion is to have only one response to a single edge. This was implemented because the first two criterions were not enough to completely eliminate the possibility of multiple responses to an edge. Canny specified three issues that an edge detector must address. They are:-

\subsubsection{Error rate}

The edge detector should respond only to edges, and should find all of them No edges should be missed.

\subsubsection{Localization}

The distance between the edge pixels as found by the edge detector and the actual edge should be as small as possible.

\subsubsection{Response}

The edge detector should not identify multiple edge pixels where only a single edge exists.

\subsection{Canny Edge Detection Technique using Morphological Operation}

The morphological operations purely deals with shapes for Digital Image Processing[22]. It is essential for image segmentation It works with two sets of images, one is the original image and the other is the structuring element called the Kernels and Masks[9-11]. The two basic morphological operations are Dilation and Erosion.

\subsubsection{Dilation}

Dilation thickens the object in an image by adding pixels to the boundaries of image its operation is highly dependent upon the shape and size of structuring elements. In the set theory, its operation can be defined as in equation (1) [12]

$$
(f \oplus b)(x y)=\max \left\{f\left(x-x^{\prime}, y-y^{\prime}\right) \mid x^{\prime} y^{\prime} \in D_{b}\right\}
$$

Where $\mathrm{f}$ is the input image, $\mathrm{b}$ is the structuring Element, $D_{b}$ Is the domain of $\mathrm{b}$ and $\mathrm{f}(\mathrm{x}, \mathrm{y})$ is assumed to equal $-\infty$ outside the domain of $f$. The structuring element is normally a flat pattern. The disk shape structuring element is used.

\subsubsection{Erosion}

Erosion makes object thin and results in reducing the object. Its operation can be defined mathematically as in. The notations have already described above.

$(f \Theta b)(x y)=\max \left\{f\left(x-x^{\prime}, y-y^{\prime}\right) \mid x^{\prime} y^{\prime} \in D_{b}\right\}$

The process of Canny edge detection show with the help of flow chart fig 1 .

\subsection{Fuzzy c-means Algorithm using Morphological Operations}

The main concern of paper was fuzzy, c-means algorithm; So standard fuzzy $\mathrm{c}$ means algorithm is used.

\subsection{Fuzzy C-Mean algorithms}

Fuzzy c-mean (FCM) clustering algorithm [15] was first introduced by Dunn [16] and was later extended by Bezdek [17]. FCM is a soft segmentation method which retains more information from input image than hard segmentation methods $[18,19,20]$. In Fzzy c-mean algorithm segmentation is done by fuzzy pixel classification, pixels are allowed to belong to multiple classes with a degree of membership between 0 and 1 [21]. It imparts flexibility to segmentation process and copes with an uncertainty factor by allowing fuzzy boundaries to exist between different clusters. It is based on minimization of the objective function equation (3).

$$
J_{m}=\sum_{i=1}^{N} \sum_{j=1}^{C} u_{i j}^{m}\left\|x_{i}-c_{j}\right\|^{2}, \quad 1 \leq m \leq \infty
$$

Where $\mathrm{m}$ is fuzzification parameter which determines the degree of fuzziness in the clusters, $u_{i j}$ Is the degree of membership of $x_{i}$ In the cluster $\mathrm{j}, x_{i}$ Is the earth data point, $c_{j}$ is the $\mathrm{j}^{\text {th }}$ cluster center, and $\|*\|$ is a squared error function.

The pseudo code for the fuzzy c-mean algorithm [15]

\subsubsection{Initialization by taking cluster centroid as random numbers.}

\subsubsection{Distance Computation}

Image is concatenated to itself along with the appropriate dimension. Cluster centroids are replicated to image dimensions and then they are concatenated to each other. The distance is computed by equation (4)

$$
\mathrm{d}_{\mathrm{ic}}=|\mathrm{I}-\mathrm{c}|
$$

Where I - input image concatenated to itself along third dimension, $\mathrm{c}$ - initial centroids replicated to image dimensions and then concatenated to each other.

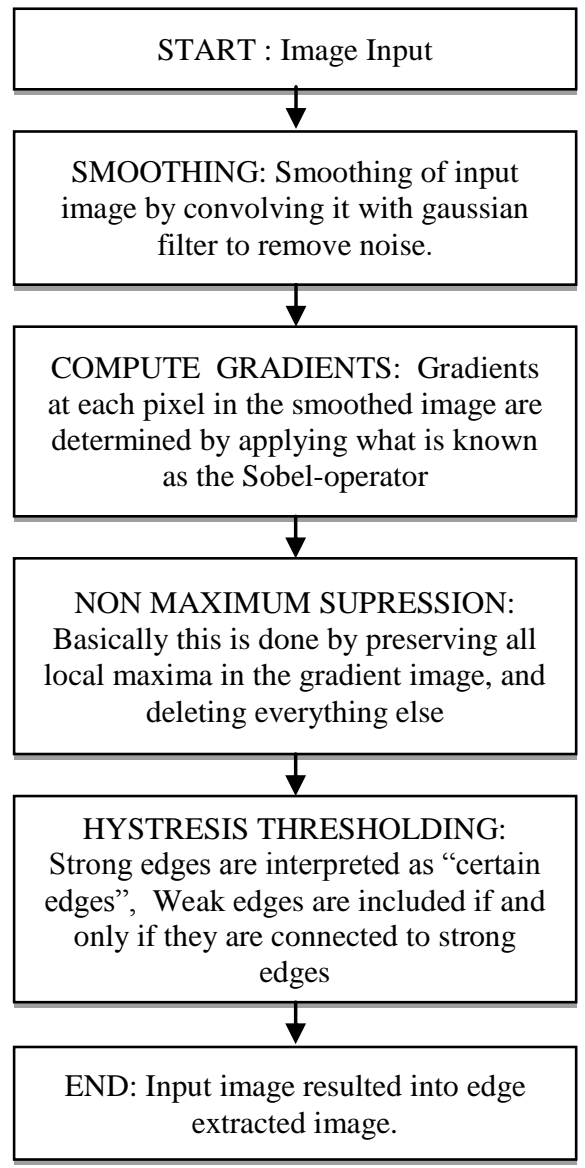

Fig1: flow chart for canny technique 


\subsubsection{Fuzzy Membership Function}

Fuzzy weight matrix is computed as the inverse of distance matrix $d_{I c}$. Then fuzzy weights are computed along first and second dimensions. Fuzzy weights define membership value of pixels into clusters. Flexibility is achieved and outliers are dealt by allowing one pixel to reside in multiple clusters.

\subsubsection{New Centroids are computed as}

Cnext1 $=\sum_{i=1}^{N}$ FuzW1 $*$ FuzW1 $* \mathrm{Ii} / \sum_{i=1}^{N}$ FuzW1 $*$ FuzW1;

Cnext2 $=\sum_{i=1}^{N}$ FuzW2 $*$ FuzW2 $* \mathrm{Ii} / \sum_{i=1}^{N}$ FuzW2 $*$ FuzW2;

\subsubsection{Stopping Criterion}

If $\max \{\mid \operatorname{cc} 1-$ Cnext $1|/ \mathrm{cc} 1,| \operatorname{cc} 2-$ Cnext2) $/ \operatorname{cc} 2 \mid\}<\varepsilon$

Then stop

\section{Otherwise}

cc1 $=$ Cnext $1 ; \quad$ cc2= Cnext $2 ;$

Go to step (2)

The image obtained by fuzzy c-means does not extract tumor properly, therefore morphological operations are applied as a post processing step to enhance results. Reconstruction based morphology $[7,23]$ applied, which is more powerful and effective tool as compare to standard morphology in eliminating the undesirable features without affecting desirable ones.

\section{RESULT}

Result define various performance parameters Percentage of detection tumor, Error, Response to the weaker edges, Minimum visibility of isolated points and Accuracy; for the comparison of these methods table (1) The result is visual, So it is more suitable to see the result given by different methods table (2).By visual observation of table (2) it is easily understand that fuzzy cmeans algorithm gives best result.

\section{CONCLUSION AND FUTURE}

work In this paper, we present different methods of detecting a brain tumor in MRI images. Conclusion shows that fuzzy cmeans clustering method gives best result as compared to the other methods. This fuzzy based method have positive points that it is less sensitive to noise, adaptive in nature and gives accurate result. So in that perception fuzzy c-means clustering method gives the exact shape of the tumor and detected all the infected areas where the tumor is found in the MRI brain image. The result of this paper is visual. So we can better understand by analyzing result visually.By varying the. technique like finding more features or changing segmentation technique the accuracy can be improved and using nueral network also classify the result obtained.

Table2. Tumor Detection Result Which We Have Found By Different Method Are Shown In This Table Y- Detected, N- Not Detected

\begin{tabular}{|c|c|c|c|}
\hline Operators & Canny(simple) & $\begin{array}{c}\text { Canny with } \\
\text { morphological operation }\end{array}$ & $\begin{array}{c}\text { Fuzzy c-means } \\
\text { clustering }\end{array}$ \\
\hline Images & $\mathrm{N}$ & $\mathrm{N}$ & $\mathrm{Y}$ \\
\hline Image1 & & & $\mathrm{Y}$ \\
\hline Image2 & & $\mathrm{Y}$ & $\mathrm{Y}$ \\
\hline & & & \\
\hline
\end{tabular}




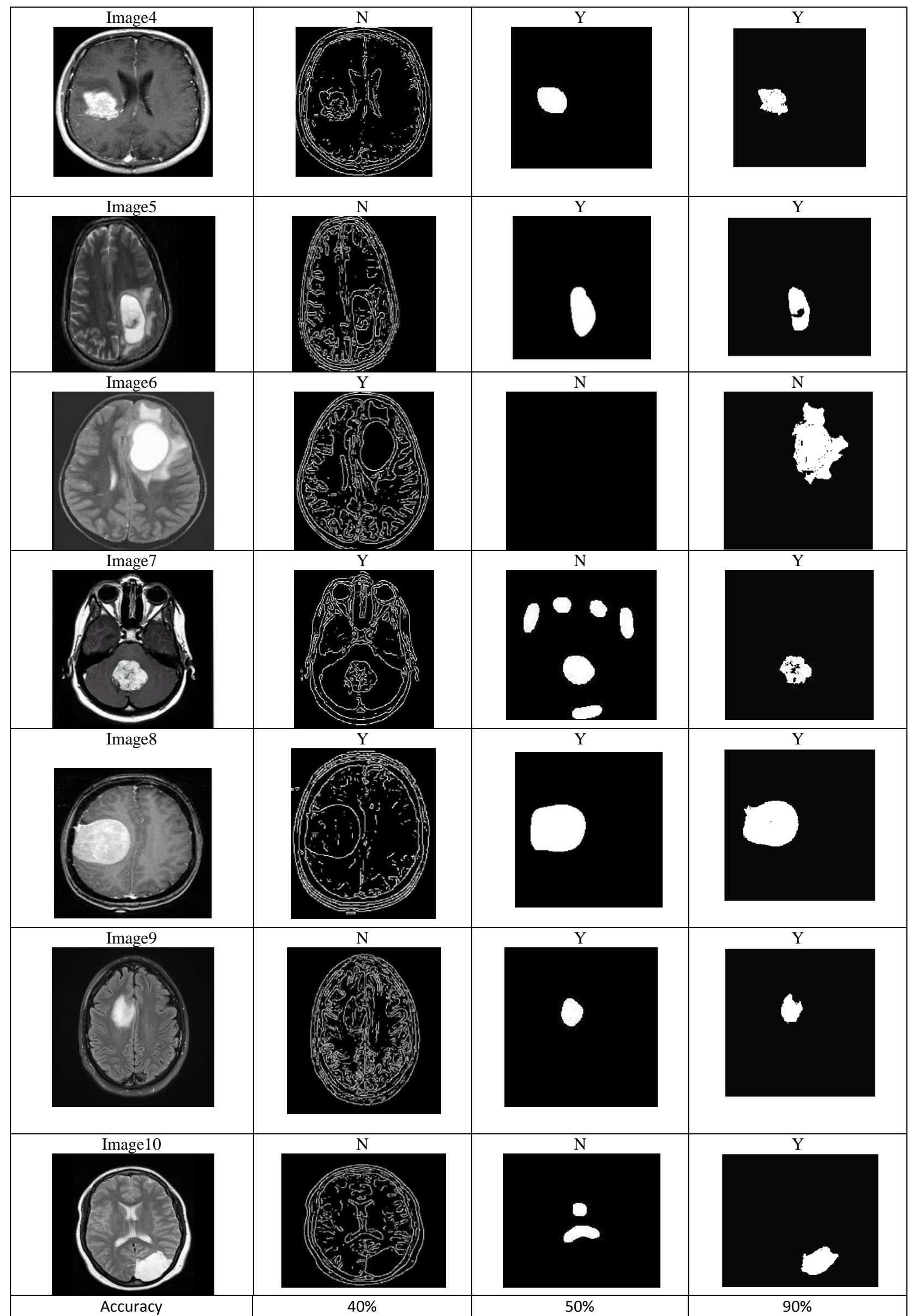


Table 1 Performance Comparison of Methods

\begin{tabular}{|c|c|c|c|}
\hline Technique & $\begin{array}{l}\text { Canny } \\
\text { edge } \\
\text { detection } \\
\text { (simple) }\end{array}$ & $\begin{array}{c}\text { Canny } \\
\text { edge } \\
\text { detection } \\
\text { using } \\
\text { mophology }\end{array}$ & $\begin{array}{l}\text { Fuzzy c- } \\
\text { means } \\
\text { algorithm } \\
\text { using } \\
\text { morphology }\end{array}$ \\
\hline $\begin{array}{l}\text { Percentage of } \\
\text { detection } \\
\text { tumor(10) }\end{array}$ & 8 & 8.5 & 9 \\
\hline $\begin{array}{l}\text { Error }=\operatorname{dim} 1- \\
\quad \operatorname{dim} 2\end{array}$ & 0.50 & 0 & 0 \\
\hline $\begin{array}{c}\text { Response to the } \\
\text { weaker } \\
\text { edges }(10)\end{array}$ & 7 & 6 & 5 \\
\hline $\begin{array}{c}\text { Minimum } \\
\text { visibility of } \\
\text { isolated points or } \\
\text { noise }(10)\end{array}$ & 8 & 9 & 8 \\
\hline Accuracy $(100 \%)$ & $40 \%$ & $50 \%$ & $90 \%$ \\
\hline
\end{tabular}

\section{CONFUSION MATRICES} All Confusion Matrix

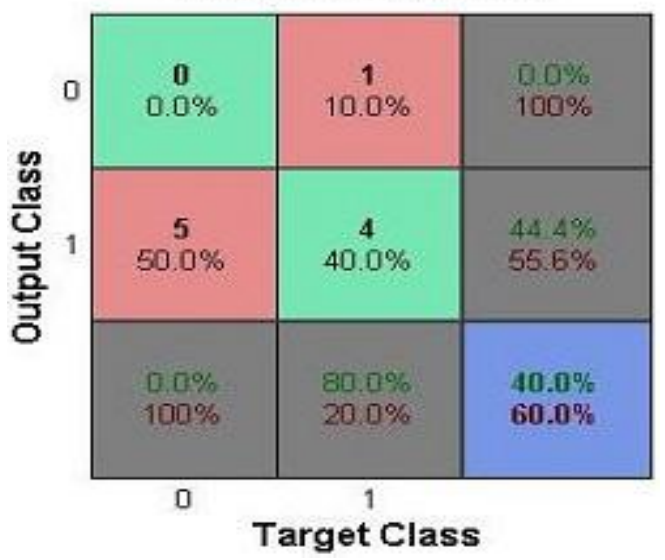

Confusion matrix for canny edge detection

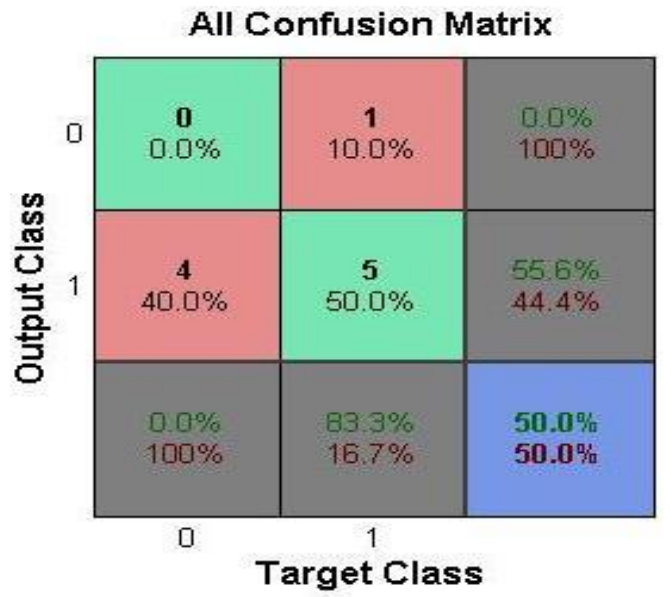

Confusion matrix for canny edge detection technique by using morphological operation

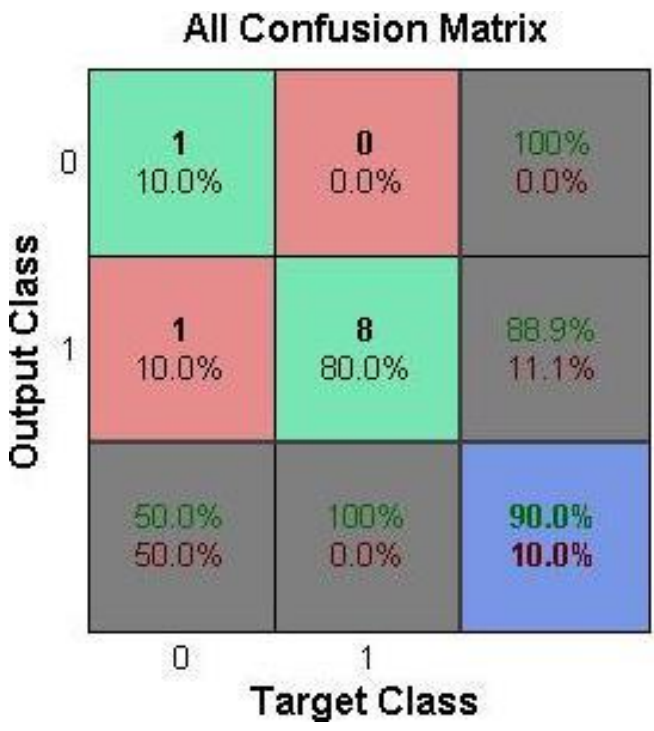

Confusion matrix for fuzzy c means technique

\section{REFERENCES}

[1] Gajanayake, Randike, Yapa, Roshan Dharshana, Hewavithana and Badra. 2009. Comparison of standard image segmentation methods for segmentation of brain tumors from $2 D$ MR images. In Proc. IEEE 4th International Conference on Industrial and Information Systems, ICIIS'09, pp. 301-305.

[2] S. M urugavalli, V. Rajamani," A high speed parallel fuzzy, c-mean algorithm for brain tumor segmentation", BIME Journal, Volume (06), Issue (1), Dec., 2006

[3] M. Rakesh, T. Ravi, "Image Segmentation and Detection of Tumor Objects in MR Brain Images Using FUZZY CMEANS (FCM) Algorithm”, IJERA, Vol. 2, Issue3, MayJun2012, pp. 2088-2094

[4] Liu Yucheng, Liu Yubin, "An Algorithm of Image Segmentation Based on Fuzzy Mathematical Morphology", International Forum on Information Technology and Applications, 401331, China 2009.

[5] M. Rastgarpour and J. Shanbehzadeh. 2011. Application of AI Techniques in Medical Image Segmentation and Novel Categorization of Available Methods and Tools. International MultiConference of engineers and computer scientists, IMECS'11, Vol. 1.

[6] A. Halder and D.K. Kola. 2012. Automatic Brain Tumor Detection and Isolation of Tumor Cells from MRI Images. International Journal of Computer Applications, Vol.39, No.2.

[7] W. A. Ulbeh, A. Mustafa and Z. A. Acadia. (2009). Gray image reconstruction. European J. Scientific Research. [Online]. 27.pp 167-173, Available: http://www.eurojournals.com/ejsr_27_2_01.pdf

[8] K. Parvati, B. S. P. Rao and M. M. Das. 2008.Image Segmentation Using Grayscale Morphology and MarkerControlled Watershed Transformation. Hindawi Publishing Corporation Discrete Dynamics in Nature and Society, Vol. 2008, Article ID 384346. 
[9] Z. Yu, Y. Zhao and X.F. Wang. 2008. Research Advances and Prospects of Mathematical Morphology in Image Processing," IEEE.

[10] L. Vincent. 1993. Morphological grayscale reconstruction in image analysis: applications and efficient algorithms. IEEE Transactions on Image Processing, Vol. 2, No. 2, pp. 176-201.

[11] C. Santhaiah, G. Anjan Babu, and M. Usha Rani. (July 2009). "Gray-level morphological operations for image segmentation and tracking edges on medical applications". IJCSNS. [Online]. 9. Pp. 131-136. Available: http://paper.ijcsns.org/07_book/200907/20090719.pdf

[12] R. Jones and I. Svalbe, "Algorithms for the decomposition of Grayscale Morphological Operation," IEEE Trans. Pattern Anal. Mach. Intell., vol. 16, pp. 581 -588, 1994.

[13] R. C. Gonzalez, R. E. Woods, "Morphological image processing," Digital Image Processing Using Matlab, Fifth Impression, Delhi, India: Pearson, 2009, ch. 9, Sec. 9.6, pp. 380-382

[14] I. Soesanti, A. Susanto, T.S. Widodo and M. Tjokronagoro. 2011. "MRI Brain Images Segmentation Based on Optimized Fuzzy Logic and Spatial Information". International Journal of Video \& Image Processing and Network Security, IJVIPNS-IJENS, Vol. 11, No.4

[15] http://www.mathworks.com/matlabcentral/fileexchange/255 32-fuzzy-c-means-segmentation
[16] J.C. Dunn. 1973. "A fuzzy relative of the ISODATA process and its use in detecting compact well separated clusters". Journal of Cybernetics, Vol. 3, No.3, pp. 32-57.

[17] J.C. Bezdek. 1981. "Pattern Recognition with Fuzzy Objective Function Algorithms", New York: Plenum Press.

[18] J. K. Udupa, L. Wei, S. Samarasekera, Y. Miki, M. A. Van Buchem, and R. I. Grossman. 1997. "Multiple sclerosis lesion quantification using fuzzy-connectedness principle". IEEE Transactions on Medical Imaging, Vol. 16, pp. 598609

[19] D.L. Pham. 2003. "Unsupervised Tissue Classification in Medical Images using Edge-Adaptive Clustering”. In Proc. 25th Annual International Conference of the IEEE EMBS, Mexico.

[20] J. Bezdek, L. Hall and L. Clarke. 1993." Review of $M R$ image segmentation using pattern recognition". Journal of Medical Physics, Vol. 20, pp. 1033-1048

[21] I. Soesanti, A. Susanto, T.S. Widodo and M. Tjokronagoro. 2011. "MRI Brain Images Segmentation Based on Optimized Fuzzy Logic and Spatial Information". International Journal of Video \& Image Processing and Network Security, IJVIPNS-IJENS, Vol. 11, No.4

[22] "Morphological operations", Image Processing Toolbox User's Guide, Aksmlore, April 07, 2013.

[23] L.Kaur and G. Kaur. 2011.Comparison of Foreground Marker control with Watershed Segmentation Algorithms for Tumor Detection in 2D MR Images. Elsevier Journal of Digital signal processing, submitted. 